\title{
Characterisation of mega-voltage electron pencil beam dose distributions: viability of a measurement-based approach
}

\author{
M. P. Barnes ${ }^{1,2}$ and M. A. Ebert ${ }^{3,4}$ \\ ${ }^{1}$ School of Mathematical and Physical Sciences, University of Newcastle, Australia \\ ${ }^{2}$ Department of Radiation Oncology, Newcastle Mater Hospital, Australia \\ ${ }^{3}$ Department of Radiation Oncology, Sir Charles Gairdner Hospital, Australia \\ ${ }^{4}$ School of Physics, University of Western Australia, Australia
}

\begin{abstract}
The concept of electron pencil-beam dose distributions is central to pencil-beam algorithms used in electron beam radiotherapy treatment planning. The Hogstrom algorithm, which is a common algorithm for electron treatment planning, models large electron field dose distributions by the superposition of a series of pencil beam dose distributions. This means that the accurate characterisation of an electron pencil beam is essential for the accuracy of the dose algorithm. The aim of this study was to evaluate a measurement based approach for obtaining electron pencil-beam dose distributions. The primary incentive for the study was the accurate calculation of dose distributions for narrow fields as traditional electron algorithms are generally inaccurate for such geometries. Kodak X-Omat radiographic film was used in a solid water phantom to measure the dose distribution of circular $12 \mathrm{MeV}$ beams from a Varian 21EX linear accelerator. Measurements were made for beams of diameter, 1.5, 2, 4, 8, 16 and $32 \mathrm{~mm}$. A blocked-field technique was used to subtract photon contamination in the beam. The "error function" derived from Fermi-Eyges Multiple Coulomb Scattering (MCS) theory for corresponding square fields was used to fit resulting dose distributions so that extrapolation down to a pencil beam distribution could be made. The Monte Carlo codes, BEAM and EGSnrc were used to simulate the experimental arrangement. The $8 \mathrm{~mm}$ beam dose distribution was also measured with TLD-100 microcubes. Agreement between film, TLD and Monte Carlo simulation results were found to be consistent with the spatial resolution used. The study has shown that it is possible to extrapolate narrow electron beam dose distributions down to a pencil beam dose distribution using the error function. However, due to experimental uncertainties and measurement difficulties, Monte Carlo is recommended as the method of choice for characterising electron pencil-beam dose distributions.
\end{abstract}

Key words electron pencil beam, radiographic film, TLD, Monte Carlo, radiotherapy

\section{Introduction}

The scattering and energy loss characteristics of electrons make accurate electron treatment planning algorithms difficult to develop. One of the most successful electron treatment planning algorithms was created by Hogstrom $(1981)^{1}$ and has since been extensively developed for clinical applications. The 'Hogstrom algorithm' utilises a superposition of pencil beam dose distributions (elemental distributions for very narrow fields) to simulate a dose

Corresponding author: Michael Barnes, Newcastle Mater Misericordiae Hospital. Department of Radiation Oncology (Physics). Locked Bag 7, Hunter Region MC, NSW, Australia Email: michael.barnes@studentmail.newcastle.edu.au Received: 10 October 2007; Accepted: 25 February 2008 Copyright (C) 2008 ACPSEM broad beam and is commonly used for electron dose calculations in radiation oncology. This concept has been extensively investigated ${ }^{1,2,3}$

The dose distribution of an electron pencil beam can be obtained theoretically with Multiple Coulomb Scattering (MCS) theory. MCS is based on the Eyges solution to the Fermi equation ${ }^{4}$ and assumes that the scattering angle of energetic charged particles traversing a medium is small. With such an approach the lateral and angular dispersion of particles undergoing multiple elastic scattering can be calculated $^{2,5}$. However, MCS has a number of inherent limitations for pencil beam dose distribution calculation. Foremost among these limitations is the inability to model range straggling of the electrons in the medium ${ }^{1}$. In practical terms, this means that pencil beam dose distributions widen slower than is predicted by $\mathrm{MCS}^{6}$. Other limitations of MCS include the inability to model large-angle scattering, delta-ray production, loss of electrons, photon production due to bremsstrahlung interactions and difficulties in incorporating the detailed 
intensity, energy and angular characteristics of electrons and photons in the beam ${ }^{7,8,9}$. In practical clinical beams from linear accelerators, the ideal pencil beam model is perturbed due to the spectral and angular characteristics of the electron beam at a patient's surface, together with the Xray contamination from accelerator components, from within the patient/phantom and from intervening air. As such, clinical implementation of pencil beam models utilise measured data extensively for empirical fitting of model parameters.

A method of empirically characterising pencil beam dose distributions is to directly measure the angular spread and hence obtain pencil beam 'kernels', This method usually involves measurement of a pencil beam produced using the pinhole technique ${ }^{7}$ or from penumbral width measurements as used by Hogstrom $(1981)^{1}$. Another empirical pencil beam characterisation method is the deconvolution method of Chui and Mohan $(1988)^{15}$. Chui and Mohan used deconvolution to extract pencil beam kernels from broad beam measurements.

Brahme et al. (1980) ${ }^{16}$ performed measurements of near-monoenergetic pencil beams from a racetrack microtron with a semiconductor detector. Such a distribution cannot be directly related to a linear accelerator where electron spectra perturbations by beam defining components and photon contamination conditions are quite different.

Accurate knowledge of the dose distribution of electron pencil beams would improve the feasibility of novel electron treatments such as intensity modulated electron therapy $^{9-11}$, electron conformal techniques and therapies involving the use of precisely machined customised bolus $^{12,13}$. It has been claimed that progress in development of such complex electron treatments has been limited by inaccuracies in electron beam dose calculations ${ }^{14}$. The principle incentive for the present study was the accurate calculation of dose distributions for narrow fields.

Monte Carlo simulation is generally considered to be the most accurate means of characterising dose distributions. Traditionally, Monte Carlo has not been used directly in treatment planning due to the clinical and economic considerations associated with the time and/or computer processing power required to conduct accurate simulations. An additional problem with Monte Carlo simulation for treatment planning is the necessity for expertise to make the best use of the code and to interpret results $^{9}$. The problems of Monte Carlo treatment planning algorithms are being solved and are now incorporated in commercial systems such as Varian Eclipse (Varian Medical Systems, Palo Alto CA) and Oncentra Masterplan (Nucletron B.V., Veenendaal, The Netherlands) that have entered the market.

In this study a novel alternative method was investigated for characterising the dose distribution of an electron pencil beam. Measurements of the dose distributions of a range of broad beams were extrapolated down to suitable beam widths for pencil beam applications. The measurement-based results were compared to calculation performed via a Monte Carlo simulation.

\section{Materials and methods}

\section{Experimental setup}

All experiments (measurements as well as simulations) involved irradiating a block of sheets of solid water with a normally incident $12 \mathrm{MeV}$ electron beam from a Varian Clinac 21EX linear accelerator (Varian Medical Systems, Palo Alto, CA). The $12 \mathrm{MeV}$ beam energy was selected arbitrarily.

Collimation was achieved with $12 \mathrm{~mm}$ thick low melting-point alloy (LMA) in a reference $15 \times 15 \mathrm{~cm}^{2}$ applicator. Circular field sizes were defined by drilling through the LMA, with resulting field diameters at isocentre of 1.5, 2, 4, 8, 16 and $32 \mathrm{~mm}$ ( $\pm 0.1 \mathrm{~mm})$. Circular fields were chosen so that radial symmetry of the dose distribution could be assumed and thus the number of required measurements was minimised. This assumption was validated by measuring the dose for a single circular field across a film in both the inplane and crossplane directions. It is also much easier to accurately drill narrow circular apertures in cutout material.

Electron pencil beams that are produced by collimating a larger beam are inevitably contaminated with bremsstrahlung photons ${ }^{17}$. To minimise this contamination, McParland investigated taking measurements with a closed collimator and then subtracting the results from the open collimator results. McParland's technique was applied to this project by taking measurements or performing simulations with a closed cutout. The closed cutout essentially provides a $0 \mathrm{~mm}$ diameter beam and such a measurement incorporates bremsstrahlung photons generated in the treatment head of the linac, in the cutout itself and in intervening air.

\section{Radiographic film measurements}

Kodak (Rochester, NY) X-Omat V film was used to measure the dose distributions of the closed cutout, 1.5, 2, 4, 8, 16 and $32 \mathrm{~mm}$ diameter beams. Film was placed in between the solid water sheets at $10 \mathrm{~mm}$ incremental depths from 0 to $80 \mathrm{~mm}$. A minimum thickness of $120 \mathrm{~mm}$ of solid water was placed below the detectors to ensure appropriate backscatter at the measurement depth. The dose at only one depth was measured per irradiation so that detectors near the surface did not perturb doses measured at greater depths $^{18}$.

Profiles along the $X$-axis were taken for each measurement using a Wellhöfer (Scanditronix-Wellhöfer, Uppsala, Sweden), optical film densitometer (sampled in 1 $\mathrm{mm}$ intervals), corrected according to a measured doseresponse curve and normalised to represent dose per linear accelerator monitor unit. Radiographic film has an associated energy dependence that has been measured to be approximately $2 \%$ for electrons with energy ranging from $0.3 \mathrm{MeV}$ to $20 \mathrm{MeV}^{19}$. As well as this, film has been known to vary in response by up to $5 \%$ from batch to batch, from film to film within a batch and even across a single film ${ }^{20}$.

\section{TLD measurements}

Due to the problems associated with film dosimetry such as energy and dose dependence ${ }^{21}$, TLD-100 
microcubes $\left(1 \mathrm{~mm}^{3}\right)$ were used as a means of verifying the film results. TLD measurements were taken for the $8 \mathrm{~mm}$ beam. TLDs were placed to provide $4 \mathrm{~mm}$ resolution in the inplane direction of with an uncertainty in the position of a TLD of $\pm 1 \mathrm{~mm}$. TLDs were calibrated to a low known dose with the $12 \mathrm{e}$ beam to avoid dose response, supralinearity and energy response. TLDs were also corrected for individual sensitivity variation and the resulting measurement uncertainty is of the order of $\pm 3 \%$.

\section{Monte Carlo simulations}

The experimental arrangement was simulated with a combination of the BEAM and EGSnrc Monte Carlo codes $^{22,23}$. Tuning of BEAM for the electron beam considered was achieved by matching simulation results to measured broad-beam data. BEAM was used to obtain electron and photon phase space information immediately above the level of the cutout. This phase space was subsequently used in a simulation of the experimental geometry using DOSXYZnrc ${ }^{23}$. Dose to water profiles at the measurement depths were obtained using 1 to $2 \mathrm{~mm}$ voxel widths. The method of photon contamination subtraction used for experimental data was similarly applied for the Monte Carlo results.

\section{Extrapolation}

A functional extrapolation of measured and simulated data was attempted in three dimensions (beam width, depth and off-axis distance). It was found that considerable noise in the measured dose profiles (of the order of $10 \%$ ) made this extrapolation unstable. An extrapolation in just two dimensions (beam width and depth) was employed instead. Noise in Monte Carlo profile results (of order $5 \%$ in the high-dose regions) was reduced by applying a moving average with a width of three voxels. This noise was despite large numbers of particle histories, and is a result of the relatively small number of phase-space particles actually passing through the very small aperture.

The convolution of a uniform circular aperture and a Gaussian distribution can be undertaken numerically, although an analytical solution has not been forthcoming. It would be possible to perform the numerical convolution directly or via Fourier methods, though this process in an iterative curve fitting would be tedious. The dose distributions from square fields for the same beam width as the circular fields were compared for a range of small field sizes and Gaussian widths, along the symmetry axis of the square fields. It was found that, within the resolution of measurement in this experiment, the resulting distributions are well within the error of these experiments, differing by less then $0.5 \mathrm{~mm}$ and only for large Gaussian widths (i.e., large depths). As an analytical solution for square fields is available, the dose distributions along a diameter for circular fields were approximated as the dose distributions along the symmetry axis of square fields of the same width. As a function of two parameters, $A$ and $B$, for a circular field onaxis with diameter $L$, the dose at a point $x$ on the $X$-axis and at depth $z$ is:
$D(L, x, z)=A(z) \cdot\left[\operatorname{erf}\left(\frac{L}{B(z)}\right)\right] \cdot\left[\operatorname{erf}\left(\frac{L+x}{B(z)}\right)+\operatorname{erf}\left(\frac{L-x}{B(z)}\right)\right],(1)$

where:

$\operatorname{erf}(\lambda)=\frac{2}{\sqrt{\pi}} \int_{0}^{\lambda} e^{-t^{2}} d t$

The parameter $A(z)$ is proportional to the depth dose for an infinitely broad field on central axis at depth $z^{24}$. The parameter $B(z)$ is proportional to the mean-square radial displacement of a Gaussian function (at depth $z$ ) representing the profile of a pencil beam at that depth. The fitting of these parameters to measured and simulated data was achieved with the Generalized Reduced Gradient optimization code included with Microsoft Excel (www.microsoft.com). A least-squares objective was employed.

\section{Results and discussion}

\section{Measurements and simulation}

Distributions of photon contamination from the treatment head and LMA material are shown as measured and simulated in Figure 1. Doses measured directly below the aperture are inaccurate using the contamination subtraction method. The contamination doses that are subtracted from the raw beam data are those that are measured below a closed cutout. This will be different to the contamination produced with a small aperture due to the different density and atomic number of air compared to the LMA used to build electron cutouts. Relative to the LMA cutout material, few bremsstrahlung photons will be produced in the air filled aperture. This means that contamination generated in the cutout is overestimated directly below the aperture. Conversely, bremsstrahlung generated in the treatment head of the linac does not have to travel through the cutout to reach the solid water. This means that photons produced in the linac treatment head will be attenuated less than photons that have to traverse the closed cutout used for contamination subtraction. As such, photons generated in the treatment head are underestimated under the aperture.

As expected figure 1 shows characteristics of photon contamination rather than electron contamination. The distribution shows a gradual drop off of dose with depth after $d_{\max }$ which is situated at or near the surface. This resembles the familiar photon depth dose curve and the $d_{\max }$ position close to or at the surface is an indication that the photon energy is in the kilovoltage range. A slight build up effect is seen in figures $1 \mathrm{~b}$ and $1 \mathrm{c}$ for film and Monte Carlo results which is not evident in TLD results of figure 1 a but the difference is likely due to the relatively large size of the microcube TLDs and their large spacing (4 $\mathrm{mm}$ in the $\mathrm{x}$-plane and $1 \mathrm{~cm}$ in the z-plane) compared to film and Monte Carlo. Profiles across figures 1a, 1b and 1c all show poor flatness which is likely due to beam softening in the 


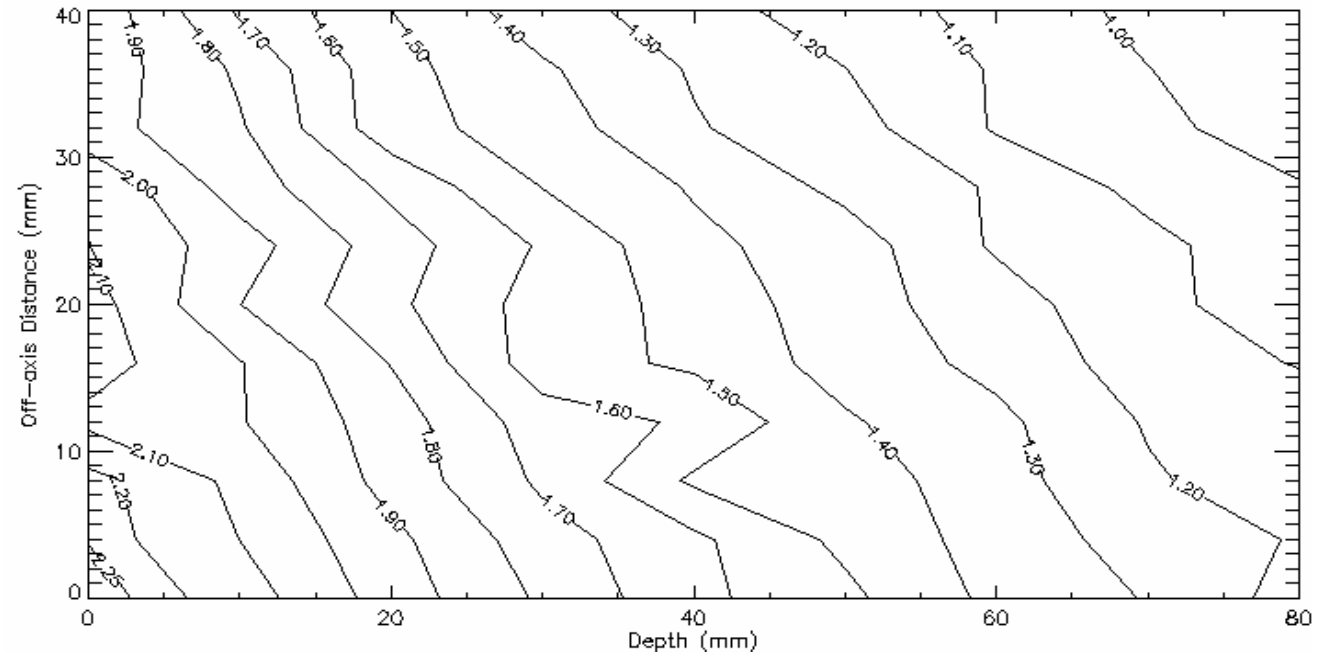

(a)

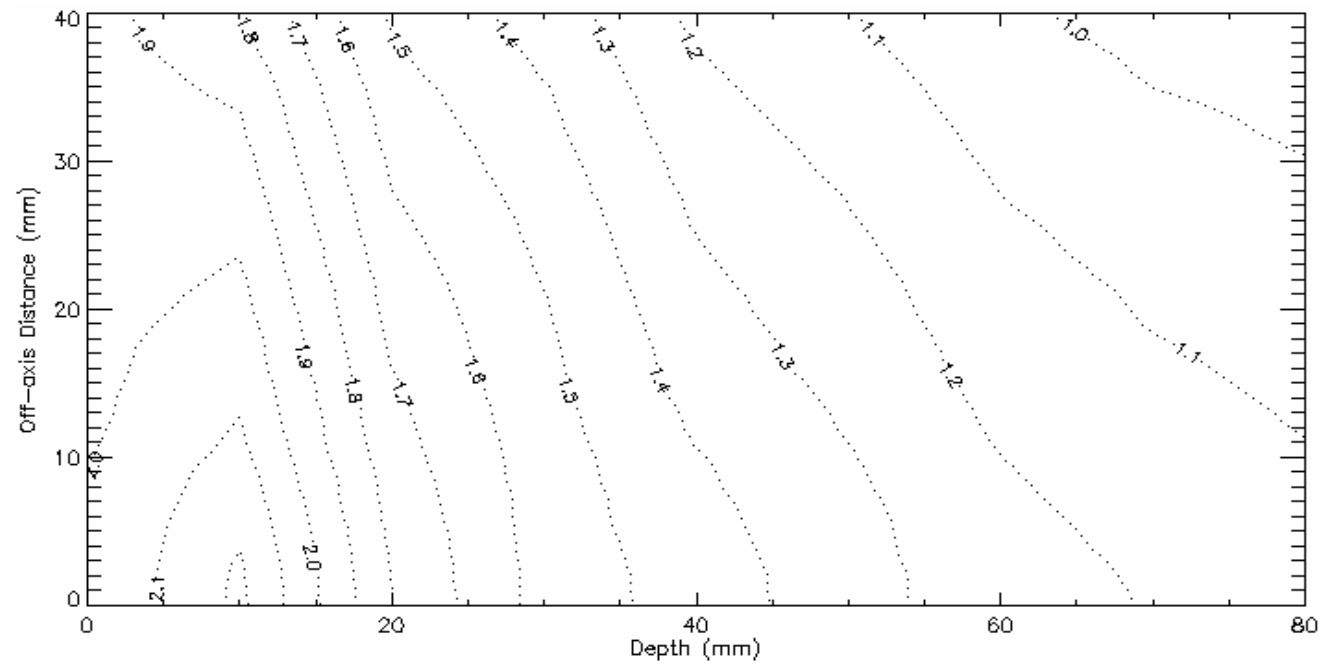

(b)

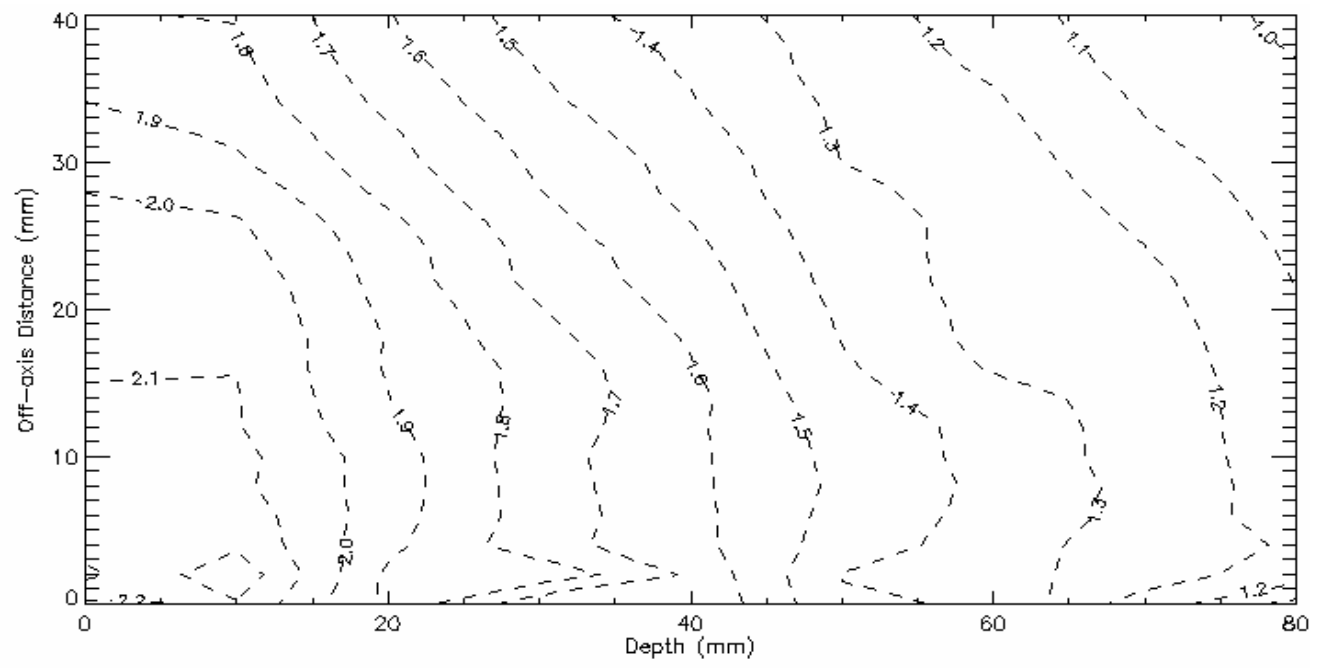

(c)

Figure 1. Dose distributions due to photon contamination in the $\mathrm{XZ}$ plane for the $12 \mathrm{MeV}$ beam. Normalisation is dose (Gy) per 100 monitor units (nominally 1 Gy at the depth of dose maximum for an open field). Results are shown for microcube TLDs, radiographic film and Monte Carlo simulation. Monte Carlo results have been smoothed due to excessive noise. All simulations utilised $2 \times 10^{7}$ phase space particles. 


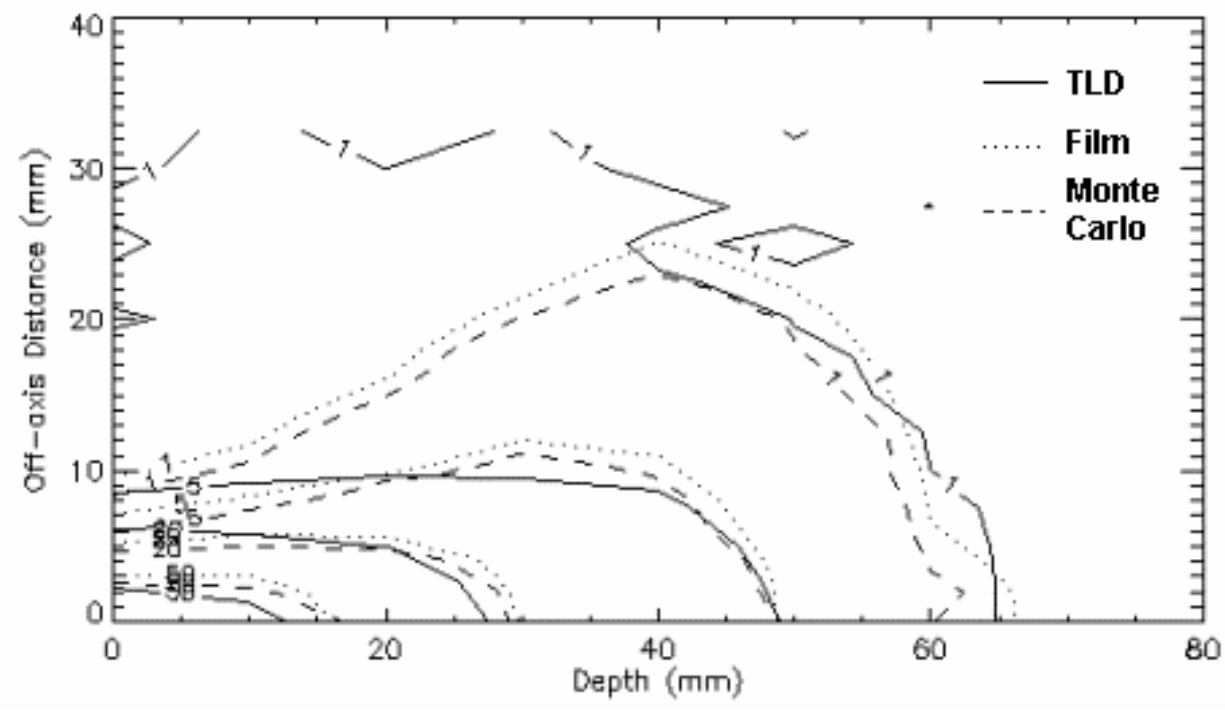

Figure 2. Dose distributions in the XZ plane for an $8 \mathrm{~mm}$ diameter beam. Isodoses are Gray (Gy) per 100 monitor units (nominally 1 Gy at the depth of dose maximum for an open field). These results include both electron and photon dose. Results shown comparing distributions obtained with radiographic film, microcube TLDs and Monte Carlo simulation.

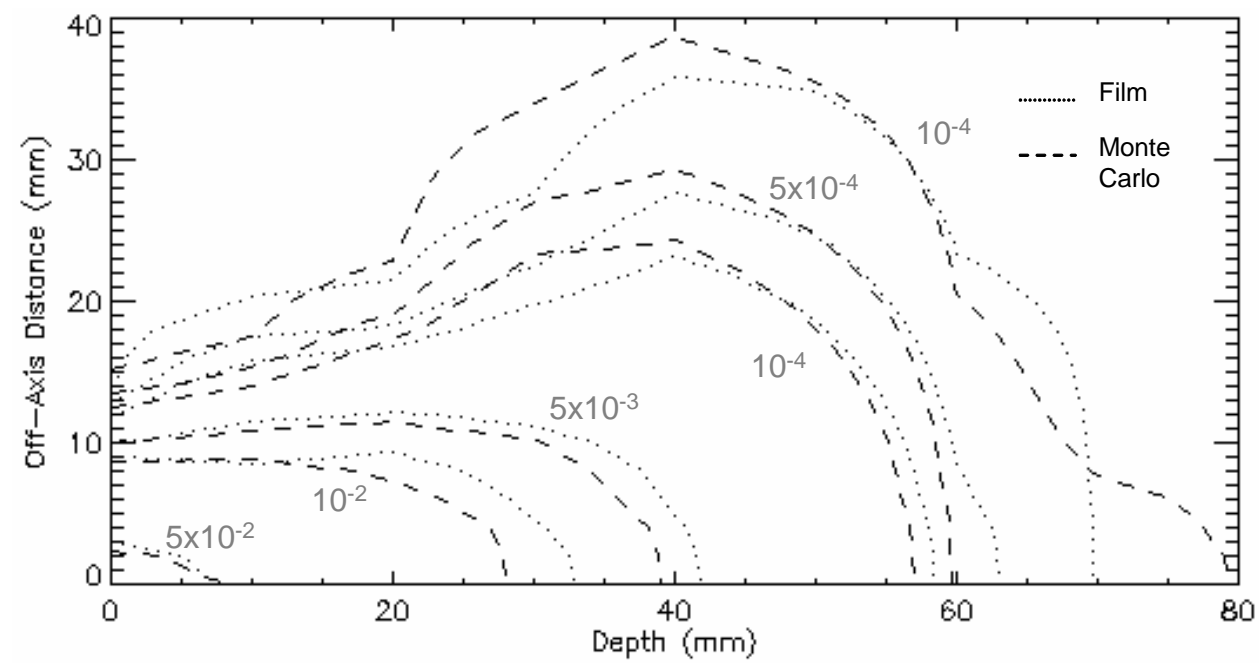

Figure 3. Extrapolated distributions for a $0.1 \mathrm{~mm}$ diameter pencil beam. Isodoses are Gy per 100 monitor units (nominally 1 Gy at the depth of dose maximum for an open field).

LMA cutout leading to a large variation in photon contamination energy across the cutout.

Dose distributions for the $8 \mathrm{~mm}$ wide beam measured with film, TLD and simulated Monte Carlo are compared in Figure 2. Resolution issues for the TLD measurements are immediately apparent, including dosimetric resolution at very low doses leading to poor definition of the lower isodose line. Otherwise, the measurement and simulation methods provided results consistent with the spatial resolution used, particularly in depth $(10 \mathrm{~mm})$. This includes agreement in absolute dose.

Whereas the spatial resolution of radiographic film would be expected to be useful in this application, dosimetric errors would be expected to be a significant disadvantage. Such errors include response energy dependence $^{19}$, non-linearity ${ }^{25}$, sensitivity variations from film to film, batch to batch and across a single film ${ }^{20}$. In combination with the necessity for contamination subtraction it was expected that film would be unreliable in this context. As such, the agreement with Monte Carlo simulation was surprising. Such agreement was consistent across all field sizes.

\section{Extrapolation}

In order to avoid sampling problems, the fitting of filmmeasured and Monte Carlo data to Equation 1 and subsequent extrapolation was undertaken at the minimum resolution of the Monte Carlo simulation (1 mm). Figure 3 


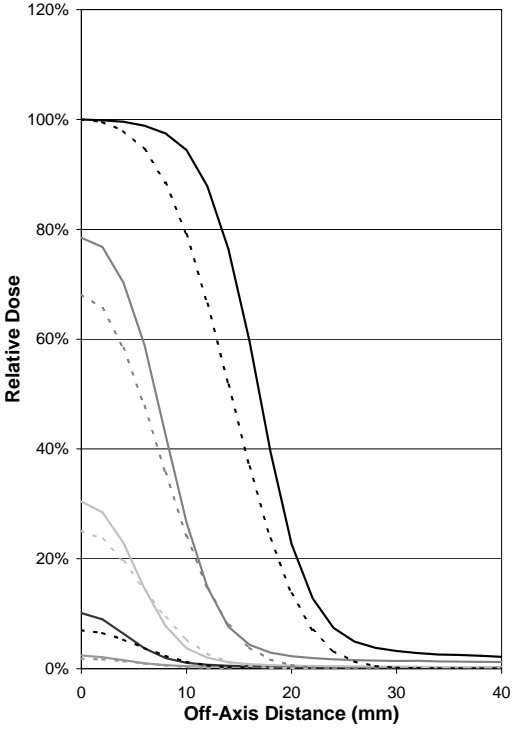

a) $20 \mathrm{~mm}$ depth, film

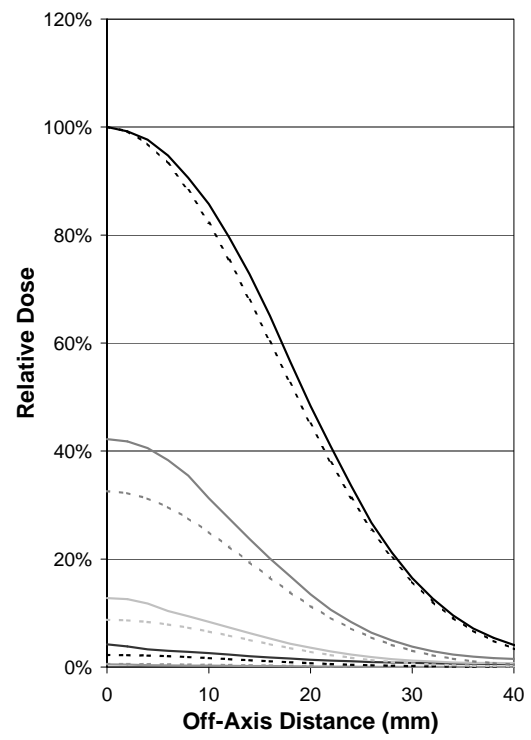

c) $50 \mathrm{~mm}$ depth, film

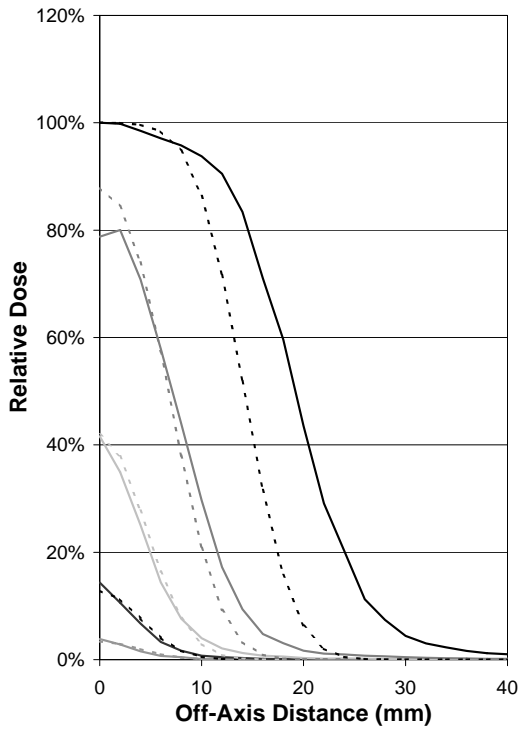

b) $20 \mathrm{~mm}$ depth, $\mathrm{MC}$

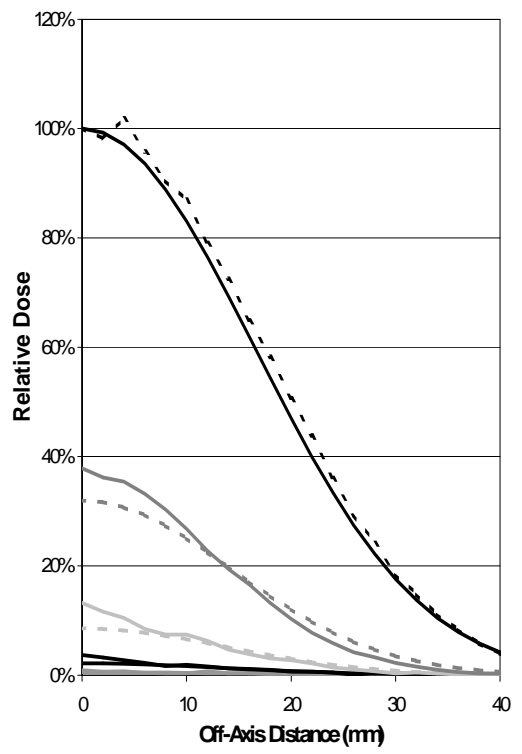

d) $50 \mathrm{~mm}$ depth, $\mathrm{MC}$

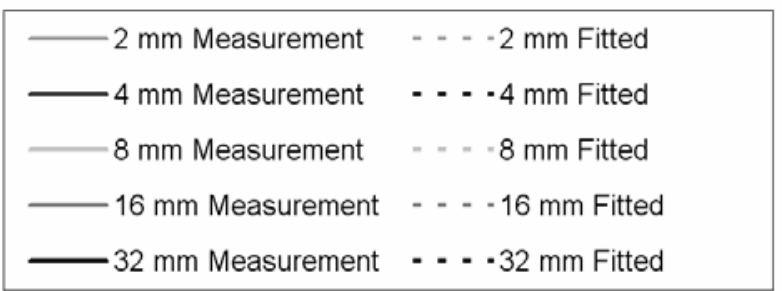

Figure 4. Comparison of measured/simulated dose distributions (profiles) and fitted dose distributions as functions of off-axis distance and field size at depths of $20 \mathrm{~mm}$ and $50 \mathrm{~mm}$.

shows the extrapolated distribution result for a field size of $0.1 \mathrm{~mm}$ diameter. No perceptible variation in the shape of the pencil beam distribution was found below this diameter.

Figure 4 provides a comparison at two depths of measured/simulated profiles and the corresponding fitted distributions from Equation 1. As can be seen, the greatest divergence occurred for the largest field size. No weighting was applied to results for any field size during the fitting procedure. It is suggested that this divergence is a result of the concentration of data points at smaller field sizes naturally weighting the curve fitting at smaller field sizes.

A recommendation from this investigation is that Monte Carlo methods are far simpler for deriving the basic dose distribution data. The methodology required for 
obtaining film-derived data is complex and resourceintensive. Although agreement with Monte Carlo has been achieved in this case, the potential uncertainties associated with using radiographic film would suggest that, if available, Monte Carlo based methods, once benchmarked, would provide more reliable data. A limiting factor with Monte Carlo is resolution, though resolution consistent with clinical radiotherapy dose calculation can be readily achieved with low statistical uncertainty.

Note that this method was primarily investigated for enhancing calculation of modulated narrow-field electron beams. Data derived from broad-beam measurements would be adequate for uniform broad-beam calculations and possibly more appropriate given the potential perturbation introduced by collimation and the fielddefining LMA for the narrow-field distributions ${ }^{6}$.

\section{Conclusions}

This investigation has shown that measured or calculated narrow-field electron beam dose distributions can be used to characterise the dose distribution from an extremely narrow (pencil beam) distribution that would not normally be possible to achieve with physical collimation. This technique would likely find benefit in application of generalised pencil beam dose calculation using pencil beam distributions, particularly for non-uniform and narrow electron fields. It would be of benefit to apply the method to square fields for which there is an analytical solution to the error function. Radial symmetry would be lost in this case and thus more measurements would be required to characterise the distribution.

Direct measurement of the beam from the accelerator, or accurate Monte Carlo simulation, allows application of a pencil beam algorithm with incorporation of the complex spectral and angular distributions of the real electron beam. Monte Carlo methods provide significant flexibility in determination of pencil beam properties. This includes opportunities for manually separating components of the primary and scattered beam, eliminating the influence of experimental design on results allowing more complete and accurate pencil beam characterisation. Monte Carlo methods are therefore recommended as the method of choice for this type of work.

\section{Acknowledgements}

The authors are grateful for the support and assistance of: Kristie Harrison, Claire Hood, Patricia Ostwald, Peter Greer, John Kenny, Kim Putré and Colin Waters for their help and input into this investigation.

\section{References}

1. Hogstrom, K. R., Mills, M. D and Almond, P. R. Electron Beam Dose Calculations. Phys. Med. Biol. 26: pp445-459, 1981.

2. Jette, D. Electron Dose Calculation using Multiple-Scattering Theory. A Gaussian Multiple-Scattering Theory. Medical
Physics. 15: pp123-137, 1988.

3. Starkschall, G., Shiu, A. S., Buynowski. S. W., Wang, L. L., Low, D. A and Hogstrom, K. A Effect of Dimensionality of Heterogeneity Corrections on the Implementation of a ThreeDimensional Electron Pencil-Beam Algorithm. Phys. Med. Biol. 36: pp207-228, 1991

4. Eyges, L. Multiple Scattering with Energy Loss. Phys. Rev. 74: pp1534-1535, 1948.

5. Klevenhagen, S. C. Physics of Electron Beam Therapy. Adam Hilger Ltd. Bristol. England, 1985.

6. Antolak, J. A., Mah, E and Scrimger, J. W. Optimisation of pencil beam widths for electron-beam dose calculations. Med. Phys. 22: pp411-419, 1995.

7. Lax, I. Inhomogeneity Corrections in Electron-Beam Dose Planning. Limitations with the Semi-Infinite Slab Approximation. Phys. Med. Biol. 31: pp879-892, 1986.

8. Jette, D. Radiation Therapy Physics; Electron Beam Dose Calculations. Springer-Verlag, Berlin. Germany, 1995.

9. Karlsson, M. G., Karlsson, M and Zackrisson, B. Intensity Modulations with Electrons: Calculations, Measurements and Clinical Applications. Phys. Med. Biol. 43: pp1159-1169, 1998.

10. Ebert, M. A and Hoban P, W. Possibilities for Tailoring Dose Distributions through the Manipulation of Electron Beam Characteristics. Phys. Med. Biol. 42: pp2065-2081, 1997.

11. Hogstrom, K. R., Antolak, J. A., Kudchadker, R. J., Ma, C. M and Leavitt, D. D. Modulated electron therapy, in Palta, J. R. and Mackie, T. R. (eds) Intensity-Modulated Radiation Therapy: The State of the Art, American Association of Physicists in Medicine Monograph 29, Medical Physics Publishing, Madison WI, 2003.

12. Low, D. A., Starkschall, G., Bujnowski, S. W., Wang, L. L and Hogstrom, K. R. Electron Bolus Design for Radiotherapy Treatment Planning: Bolus Design Algorithms. Med. Phys. 19: pp115-24, 1992.

13. Kudchadker, R. J., Hogstrom, K. R., Garden, A. S., McNeese, M. D., Boyd, R. A and Antolak J. A. Electron Conformal Radiotherapy Using Bolus and Intensity Modulation. Int. J. Rad. Oncol. Biol. Phys. 53: pp1023-1037, 2002.

14. Fraass, B. A., Smathers, J and Deye, J. Summary and Recommendations of a National Cancer Institute Workshop on Issues Limiting the Clinical use of Monte Carlo Dose Calculation Algorithms for Megavoltage External Beam Radiation Therapy. Medical Physics. 30: pp3206-3216, 2003.

15. Chui, C. S and Mohan, R. Extraction of Pencil Beam Kernels by the Deconvolution Method._Med. Phys. 15: pp138-144, 1988.

16. Brahme, A., Kraepelien, T. and Svensson, H. Electron and photon beams from a $50 \mathrm{MeV}$ racetrack microtron. Acta Radiol. Oncol. 19: pp305-319, 1980.

17. McParland, B. J. Using Background Subtraction for Measuring the Dose Distribution of an Electron Pencil Beam. Med. Phys. 14: pp406-409, 1986.

18. Suchowerska, N., Hoban, P., Davison, A and Metcalfe, P. Perturbation of Radiotherapy Beam by Radiographic Film; Measurements and Monte Carlo Simulations. Phys. Med. Biol. 44: pp1755-1765, 1999.

19. Dutreix, J and Dutreix, A. Film Dosimetry of High Energy Electrons. Ann. NY. Acad. Sci. 161: pp33-43, 1969.

20. Khan, F. M., Doppke, K. P., Hogstrom, K. R., Kutcher, G. J., Nath, R., Prasad, S. C., Purdy, J. A., Rozenfeld. M and Werner, B. L. Clinical Electron-Beam Dosimetry: Report of AAPM Radiation Therapy Committee Task Group No. 25. Med. Phys. 18: pp73-109, 1991.

21. McKinlay, A. F. Thermoluminescence Dosimetry._Adam Hilger Ltd. Norwich, England, 1981. 
22. Rogers, D. W. O., Faddegon, B. A., Ding, G. X., Ma, C. M and Wei, J. BEAM: A Monte Carlo Code to Simulate Radiotherapy Treatment Units. Med. Phys. 22: pp503-524, 1995.

23. Kawrakow, J and Rogers, D. W. O. The EGSnrc Code System: Monte Carlo Simulation of Electron and Photon Transport. Technical Report PIRS-701. National Research Council of Canada. Ottawa, Canada, 2000.

24. Khan, F. M. Dose Distribution Algorithms for Electron Beams, in Khan, FM. and Potish, RA. (eds). Treatment Planning in Radiation Oncology, Lippincott Williams and
Wilkins, Philadelphia PA, 2000.

25. Van Battum, L. J and Huizenga, H. Film Dosimetry of Clinical Electron Beams. Int. J. Rad. Oncol. Biol. Phys. 18: pp69-76.

26. Ebert, M. A and Hoban P, W. A Monte Carlo Investigation of Electron Beam Applicator Scatter. Med. Phys. 22: pp14311435, 1995.

27. Korevaar, E. W., Huizenga, H., Lof, J., Stroom, J. C., Leer, J. $\mathrm{W}$. $\mathrm{H}$ and Brahme, A. Investigation of the Added Value of High-Energy Electrons in Intensity-Modulated Radiotherapy: Four clinical cases. Int. J. Radt. Oncol. Biol. Phys. 52: pp236-253, 2002. 